\title{
Commentary
}

\section{Anemia, iron deficiency and malaria amidst impoverishment: a nexus for the anemia management challenge}

\author{
Sabuktagin Rahman ${ }^{1}$, Santhia Ireen ${ }^{2}$, Nazma Shaheen ${ }^{3}$ \\ ${ }^{1}$ Griffith University, Public Health, School of Medicine, Gold Coast Campus, QLD 4222, Australia \\ ${ }^{2}$ Alive and Thrive, FHI360, Bangladesh; Gulshan 2, Dhaka, Bangladesh \\ ${ }^{3}$ Institute of Nutrition and Food Science, University of Dhaka; Dhaka, Bangladesh
}

\begin{abstract}
*Corresponding author
Sabuktagin Rahman

Griffith University, Public Health, School of Medicine, Gold Coast Campus, QLD 4222, Australia email: rahman.sabuktagin@gmail.com
\end{abstract}

We have read the article "Prevalence of Anemia, Iron-Deficiency Anemia, and Associated Factors among Children Aged 1-5 Years in the Rural, Malaria-Endemic Setting of Popokabaka, Democratic Republic of Congo: A Cross-Sectional Study" of Mbunga et al with great interest (Mbunga et al., 2021). The context and the findings - the high prevalence of childhood anemia $(68.1 \%)$ and malaria (86.8\%) and the low level of iron deficiency (ID) (1.8-12.9\%, according to the different methods) in an impoverished rural setting of the Democratic Republic of Congo posit a challenge for controlling the high burden of anemia. We agree to the explanations of the authors regarding the genesis of high prevalence of anemia; however, we explain below how it is very difficult to estimate the prevalence of ID in a setting where there is a high burden of malaria. Therefore, we offer supplementary information for the elucidation of the ID and anemia prevalence in the context of malarial endemicity. Additionally, we propose a modification in the management of the high anemia in this kind of setting.

We assume that hepcidin, the master regulator of iron metabolism (Ganz et al., 2012), possibly played the central role. Hepcidin is activated in a state of infection, inflammation and/or in the actual good status of iron in the body (Spottiswoode et al., 2014). Hepcidin is upregulated in malaria in symptomatic and asymptomatic natural human infections (Howard et al., 2007; de Mast et al., 2010). The upregulation inhibits absorption of dietary iron, causes iron to accumulate in macrophages and reducing serum iron, possibly contributing to the dyserythropoiesis and anemia 
that may accompany malaria infections (Prentice et al., 2012). Though unmeasured in the Mbunga et al study, the overwhelming prevalence of malaria suggests that there might have been a good level of circulating hepcidin in the study children which complements the high prevalence of anemia. The presence of food insecurity, low dietary diversity, low animal food intake and poverty (Mbunga et al., 2021) entail that the iron from the dietary sources was suboptimum, thus further augmenting the proposition of acquired dyserthopoiesis. Additionally, the other possible cause of the anemia could be the malarial parasite-induced premature phagocytosis and destruction of the red blood cells by the reticuloendothelial system (Perkins et al., 2011).

The infection-adjusted ID was low according to the low level of serum ferritin $(1.8 \%<12 \mu \mathrm{g} / \mathrm{L})$ and the transferrin saturation (12.9\%). The reported iron supplementation in the preceding 3 months of only in $35 \%$ of the children, is unlikely to result in such a low level of ID. On account of the high prevalence of malaria, serum ferritin was increased markedly as an acute-phase reactant to contain the infection, concomitant with a high proportion $(\sim 50 \%)$ of the elevated C- Reactive Protein (CRP). Contrary to the expected outcome, hemoglobin was not correlated with transferrin saturation. However, it presented a weak, negative correlation with ferritin. Further, hemoglobin was negatively correlated with CRP, signifying a plausible role of infection/inflammatory conditions (i.e. malaria) in the pathophysiology of the anemia.

In theory, adjustment of ferritin to exclude for the elevated acute phase reactants and to isolate the true ID can be done as per the internal regression method (Namaste et al., 2017). However, in the present case, applying this adjustment might not have assured that sufficient circulating iron was available for the heme synthesis according to the hepcidin based redistribution of iron (i.e. from serum to macrophage). In addition, Castberg et al. (2018) reported that in a malaria endemic setting, the CRP values in children were normalized at least 2 weeks earlier than the serum ferritin. They suggested that the internal regression correction method which is based on the linear association of CRP and ferritin, may not be adequate to fully compensate for the effect of malaria on ferritin; and thus could be unreliable to accurately quantify ID in a malaria endemic setting.

Although studies regarding hepcidin and malaria have mostly reported an upregulation of hepcidin in malaria infection, in certain contexts, hepcidin suppression may occur, especially in cases with severe malaria (Casals-Pascual et al., 2012) or in cerebral malaria (Burté et al., 2013). The mechanisms of hepcidin suppression could be by erythropoietic drive, hypoxia, or iron deficiency (Spottiswoode et al., 2014). Since the prevalence of malaria was exceedingly high in the setting (Mbunga et al., 2021), we assume that there might be a proportion of the cases with severe malarial infection leading to possible hepcidin suppression. Hence, the ID might be higher than that reported in the study as a possible consequence of hepcidin suppression. .

Summarising this, although it is unknown as to which way the hepcidin responded (as it was unmeasured), one possibility is that it was upregulated, thereby inducing the redistribution of serum iron, conceivably causing a state of ID; and the magnitude of ID might thus be higher than that reported in the study. At the other extreme, in case of the down-regulation of hepcidin (among the cases with severe malaria), ID could be tentatively linked with accelerated erythropoiesis. Either way, the true prevalence of ID could be plausibly underestimated in the study. The current strategies of adjustment of ferritin for the infection, one of which was used in the Mbunga et al study (i.e. regression correction), are unlikely to be accurate in the malaria-endemic context. 
The authors correctly recommended infection (i.e. malaria) control as the measure for the anemia control in this setting. However, with the likelihood of functional deficiency of the hemopoetic iron, this can be complemented by dietary and/or seasonal or targeted supplemental iron interventions which have documented benefits, even in malaria-prone settings (Spottiswoode et al., 2014). Some authors also maintaining that a protocol for malaria management in tandem with context-feasible seasonal (Atkinson et al., 2014) and/or targeted (Pasricha et al., 2014) supplements of iron and other hemopoietic nutrients can be the mainstay of the management; especially in an impoverished setting with low availability of high bioavailable dietary iron.

Author's contribution: S.R.: Conceptualization of the idea and wrote the first draft; N.S. and S.I.: Reviewed and edited to finalize. All the authors have read the final version and approved.

Conflicts of Interest Statement: The authors declare no competing interest.

\section{References}

Atkinson S. H., Armitage A. E., Khandwala S., Mwangi T. W., Uyoga S., Bejon P. A., et al. 2014. Combinatorial effects of malaria season, iron deficiency and inflammation determine plasma hepcidin concentration in African children. Blood 123(21):3221-9. DOI: 10.1182/blood-2013-10-533000

Burté F., Brown B.J., Orimadegun A.E., Ajetunmobi W.A., Afolabi N.K., Akinkunmi F.,et al. 2013. Circulatory hepcidin is associated with the anti-inflammatory response but not with iron or anemic status in childhood malaria. Blood 121(15):3016-22.

Casals-Pascual C, Huang H, Lakhal-Littleton S, Thezenas ML, Kai O, Newton CR, et al. 2012. Hepcidin demonstrates a biphasic association with anemia in acute Plasmodium falciparum malaria. Haematologica 97(11):1695-8.

Castberg, F.C., Sarbah, E.W., Koram, K.A. et al. 2018. Malaria causes long-term effects on markers of iron status in children: a critical assessment of existing clinical and epidemiological tools. Malar J 17:464. https://doi.org/10.1186/s12936-018-2609-6

de Mast Q., Syafruddin D., Keijmel S., Riekerink T. O., Deky O., Asih P. B., et al. 2010. Increased serum hepcidin and alterations in blood iron parameters associated with asymptomatic P. falciparum and $\mathrm{P}$. vivax malaria. Haematologica 95:1068-1074. DOI: 10.3324/haematol.2009.019331

Ganz, T.; Nemeth, E. 2012. Hepcidin and iron homeostasis. Biochim Biophys Acta 1823:1434-1443.

Howard C. T., McKakpo U. S., Quakyi I. A., Bosompem K. M., Addison E. A., Sun K., et al. 2007. Relationship of hepcidin with parasitemia and anemia among patients with uncomplicated Plasmodium falciparum malaria in Ghana. Am J Trop Med Hyg 77:623-626 
Mbunga, B.K.; Mapatano, M.A.; Strand, T.A.; Gjengedal, E.L.F.; Akilimali, P.Z.; Engebretsen, I.M.S. 2021. Prevalence of Anemia, Iron-Deficiency Anemia, and Associated Factors among Children Aged 1-5 Years in the Rural, Malaria-Endemic Setting of Popokabaka, Democratic Republic of Congo: A Cross-Sectional Study. Nutrients 13:1010. https://doi.org/10.3390/nu13031010

Namaste, S.M.L.; Rohner, F.; Huang, J.; Bhushan, N.L.; Flores-Ayala, R.; Kupka, R.; et al. 2017. Adjusting ferritin concentrations for inflammation: Biomarkers Reflecting Inflammation and Nutritional Determinants of Anemia (BRINDA) project. Am J Clin Nutr 106(Suppl. 1):359S-371S.

Pasricha S. R., Atkinson S. H., Armitage A. E., Khandwala S., Veenemans J., Cox S. E., et al. 2014. Expression of the iron hormone hepcidin distinguishes different types of anemia in African children. Sci Transl Med 6(235):235re3. DOI: 10.1126/scitranslmed.3008249

Perkins D.J., Were T., Davenport G.C., Kempaiah P., Hittner J.B., and Ong'echa J.M. 2011. Int J Biol Sci 7(9):1427-1442. doi: 10.7150/ijbs.7.1427Spottiswoode, N., Duffy, P. E., \& Drakesmith, H. 2014. Iron, anemia and hepcidin in malaria. Frontiers in Pharmacology 5:125. https://doi.org/10.3389/fphar.2014.00125

Prentice A. M., Doherty C. P., Abrams S. A., Cox S. E., Atkinson S. H., Verhoef H., et al. 2012. Hepcidin is the major predictor of erythrocyte iron incorporation in anemic African children. Blood 119:1922-1928. DOI: 10.1182/blood-2011-11-391219

Spottiswoode, N., Duffy, P. E., \& Drakesmith, H. 2014. Iron, anemia and hepcidin in malaria. Frontiers in Pharmacology 5:125. https://doi.org/10.3389/fphar.2014.00125 